\title{
ÉCART ENTRE LA NORME ET L'USAGE : LE CAS DU FINNOIS
}

\author{
Jukka HAVU \\ Université de Tampere, Finlande
}

\begin{abstract}
En): Finland was a Swedish province from the 13th century until 1809, when it was annexed by the Russian Empire as an autonomous Grand Duchy. In spite of the annexation, the country preserved its administrative and legislative institutions. In connection to the new situation, it became necessary to codify the Finnish language, which from 1809 was the mother tongue of $85 \%$ of the population. The sources for the codification process were several: old literary language, different regional dialects, borrowed foreign words, etc. The result was a supradialectal norm, not spoken by anybody spontaneously but accepted by all. One of the consequences of this is a considerable gap between the standard spoken Finnish and the normative language.
\end{abstract}

Keywords (En): Finnish language; codification; norm; usage.

Mots-clés (Fr): Langue finnoise ; codification ; norme ; usage.

\section{Brève introduction à l'histoire de la Finlande}

Pour comprendre l'évolution et la codification de la langue finnoise, il est utile de connaître les grandes lignes de l'histoire politique du pays, marquée par plusieurs siècles de domination étrangère (v. JUTIKKALA, 1978, HENTILÄ et al., 1999, LE CALLOC'H, 2010). Au $12^{\mathrm{e}}$ siècle, les Suédois entreprirent la conquête des territoires qui aujourd'hui correspondent à l'État de Finlande. Jusqu'en 1809, pendant plus de six siècles, la Finlande fut une province suédoise tout en conservant, pourtant, une idiosyncrasie clairement différente de la Suède proprement dite. En 1808, le tsar Alexandre I envahit la Finlande pour forcer la Suède à appliquer le blocus continental décrété par Napoléon. Le résultat de l'invasion fut l'annexion de la Finlande à l'Empire russe ; lors de la diète de Porvoo en 1809 le tsar accorda le statut de Grand-Duché autonome au pays nouvellement conquis et s'engagea à respecter « ses lois fondamentales », à savoir ses institutions administratives héritées de l'époque suédoise. La Finlande était représentée auprès de la cour impériale par le secrétaire d'État pour la Finlande, qui soumettait les décisions du Sénat finlandais (dont les membres étaient tous des Finlandais) à l'approbation du Tsar Grand-Duc.

Le rapport entre l'Empire et la Finlande fut donc celui d'une union personnelle, matérialisée dans la personne du tsar; les Russes n'avaient pas automatiquement la citoyenneté finlandaise et la langue russe n'avait pas de statut officiel en Finlande. Tous les tsars russes, jusqu'à Nicolas II, renouvelèrent l'acte de garantie qui assurait le maintien en vigueur de la Constitution du Grand-Duché. Pourtant, en 1899, le rapport entre la Finlande et l'Empire se détériora à cause des tendances panslavistes répandues en Russie. Le tsar Nicolas II émit le «manifeste de février », où il supprimait le statut relativement indépendant des institutions finlandaises en décrétant une nouvelle procédure législative qui devait être observée sur tout le territoire de l'Empire, la Finlande incluse. Le Gouverneur 
général de la Finlande, le général Bobrikoff, fut chargé d'appliquer le manifeste, considéré comme le coup d'envoi des campagnes de russification.

La résistance des Finlandais fut massive. Le général Bobrikoff fut assassiné en 1904, et, en raison des troubles internes de la Russie, le tsar dut abroger (ou plutôt suspendre) la procédure instituée par le manifeste de février. Une réforme parlementaire fut mise en place en 1906. Les premières élections au suffrage universel et secret eurent lieu en 1907 et pour la première fois dans le monde, les pleins droits électoraux (y compris l'éligibilité) furent accordés aux femmes. La campagne de russification reprit de plus belle en 1908, mais la Révolution de février mit fin au pouvoir autocratique des Tsars et celle d'octobre instaura le régime bolchévique. Les Finlandais décidèrent de proclamer l'indépendance du pays le 6 décembre 1917.

L'époque d'autonomie (1809-1917), malgré les tensions du début du $20^{\mathrm{e}}$ siècle, fut marquée par de grands progrès dans presque tous les domaines de la société. Cela était dû essentiellement au statut presque indépendant de la Finlande, placée « désormais au rang des nations », selon l'expression d'Alexandre $1^{\text {er }}$ dans son discours de clôture à la Diète de Porvoo du 27 mars 1809. Le destin du pays était désormais dans les mains des Finlandais; la formule attribuée à A. I. Arwidsson (1791-1858), un des premiers nationalistes militants de la Finlande autonome : «Nous ne sommes plus Suédois, nous ne voulons pas devenir Russes, soyons donc Finlandais! », décrit bien les conditions dans lesquelles démarre le travail de modernisation du pays après 1809 (RANTANEN, 1998 : 146).

\section{La langue finnoise}

Aujourd'hui, le finnois, langue finno-ougrienne, est la première langue nationale de la Finlande, parlée par environ $93 \%$ de la population totale du pays (environ 5 millions de locuteurs). Il y a des minorités finnophones en Suède (selon l'enquête de 2005, quelque 450.000 personnes déclarent parler ou du moins comprendre le finnois), en Norvège (entre 10.000 et 15.000 locuteurs), en Russie environ 20.000 locuteurs. Il y a aussi des communautés de finnophones aux ÉtatsUnis, au Canada et en Australie, mais dans ces pays la langue ne se transmet normalement plus des parents aux enfants (v. JÖNSSON-KORHOLA\&LINDGREN, 2003).

Comme toutes les langues finno-ougriennes, le finnois est une langue agglutinante et possède une morphologie relativement complexe. Le système nominal se caractérise par un nombre assez élevé de cas (au total 14) et par l'absence d'articles et de genre; le système verbal, qui est du type passé vs. nonpassé (il n'y a pas de futur morphologique), contient un grand nombre de formes nominales (infinitifs et participes). Ces quelques observations sur la structure du finnois ont pour but de donner une idée très approximative des différences qui séparent cette langue des langues indo-européennes.

\subsection{La langue finnoise pendant l'époque suédoise}

Jusqu'à la fin du $16^{\mathrm{e}}$ siècle, la langue finnoise était parlée par toutes les couches de la société. Au siècle suivant, la Suède devint une grande puissance politique et militaire et l'aristocratie se transforma petit à petit en une aristocratie 
de cour et adopta le suédois comme seule langue de culture. La bourgeoisie imita les pratiques langagières de la noblesse d'où la dichotomie suédois - langue de culture, finnois - langue plébéienne, qui dura pendant une bonne partie du $19^{\mathrm{e}}$ siècle.

Des premiers siècles de domination suédoise, il ne reste pas de documents écrits en finnois. Les premiers textes apparaissent dans les années 1540 grâce à l'implantation de la Réforme protestante dans le Royaume de Suède. Mikael Agricola, important réformateur et «père de la littérature finlandaise », rédigea le premier abécédaire finnois, Abckiria (en 1540), traduisit le Nouveau Testament à partir de la version grecque d'Erasme en 1548 et publia d'autres textes religieux entre 1549-1552. La Bible entière ne fut traduite qu'en 1642, presque un siècle après la publication de la traduction du Nouveau Testament (ce qui met en évidence la décadence culturelle des pays du nord après le triomphe de la Réforme).

En plus des textes religieux, une bonne partie des documents juridiques et administratifs furent traduits en finnois, entre autres la Loi du Roi Christophe (1548), les différentes lois générales du Royaume de Suède (1601, 1609, 1618), conservées en manuscrits, la Loi ecclésiastique (1688), et finalement le grand Code civil de 1734 (la traduction finnoise date de 1759). En outre, plusieurs lettres, décrets et arrêtés royaux furent publiés en finnois pendant les $16^{\mathrm{e}}, 17^{\mathrm{e}}$ et $18^{\mathrm{e}}$ siècles.

La première grammaire imprimée du finnois fut celle d'Aeschillus Petraeus, Linguae Finnicae Brevis Institutio (PETRAeus, 1649). La description de l'auteur suit les modèles gréco-latins, et les différents cas grammaticaux du finnois étaient réduits à six catégories selon le modèle latin. Cette façon de présenter la déclinaison des noms fut aussi celle de Matthias Martinus, Hodegus Finnicus (MARTINUS, 1689). En revanche, la Grammatica Fennica par Bartholdus Vhaël (VHAËL, 1733) marque un progrès considérable en adoptant comme base de la description de la langue les structures propres de la langue finnoise. Le premier dictionnaire important fut celui de Daniel Juslenius, Suomalaisen Sana-Lugun Coetus/Fennici Lexici Tentamen, (JUSLENIUS, 1745), un dictionnaire finnois-latinsuédois, avec environ 19.000 entrées.

Le finnois était utilisé presque uniquement à des fins religieuses et administratives; la littérature proprement dite n'apparâit qu'au $19^{\mathrm{e}}$ siècle. Il semble évident, pourtant, que le finnois avait un statut semi-officiel dans le royaume. Plusieurs aspects sociolinguistiques le prouvent : i) il y avait un poste de traducteur officiel auprès de la chancellerie royale de Suède ; ii) à partir du $17^{\mathrm{e}}$ siècle, une connaissance suffisante du finnois était exigée des fonctionnaires de l'État nommés aux charges publiques dans les régions finnophones du pays ; iii) la langue de culte était le finnois dans toutes les paroisses finnophones du pays ; iv) à Stockholm, la paroisse finlandaise avait été fondée en 1533 et vers le début du $17^{\mathrm{e}}$ siècle, elle adopta le finnois comme seule langue de prédication; v) les Rois de Suède avaient un professeur de finnois depuis l'époque de Gustave Vasa (vers le milieu du $16^{\mathrm{e}}$ siècle), etc.

Le traité de paix avec la Russie de 1721 marque le début du déclin politicomilitaire de la Suède, qui dut céder des territoires assez étendus à la Russie. Les 
premiers mouvements séparatistes et un timide nationalisme culturel apparaissent en Finlande vers la fin du $18^{\mathrm{e}}$ siècle. H. G. Porthan (1739-1804), «père de l'historiographie finlandaise », montra que les Finnois constituaient le peuple autochtone du pays, ce qui contribua à faire naître une nouvelle conscience de la particularité ethnique et linguistique de la Finlande.

Au seuil du grand changement politique survenu en 1809, pourtant, le finnois était une langue minoritaire, parlée par un peu moins de $25 \%$ de la population totale du Royaume (v. MEINANDER, 2008). C'était aussi une langue culturellement et socialement minorisée, surtout à partir du début du $17^{\mathrm{e}}$ siècle. Or, grâce aux tendances romantiques, à la fin du $18^{\mathrm{e}}$ siècle, apparut un nouvel intérêt pour la langue finnoise et pour les traditions de la population finnophone.

\subsection{La langue finnoise pendant l'époque autonome}

Au lendemain du Traité de Paix de Hamina (1809), ${ }^{1}$ la situation linguistique apparut certainement presque intacte aux habitants de la Finlande. Or, en réalité, tout avait changé. Même si le suédois restait la seule langue officielle du Grandduché, la population finnophone constituait dorénavant $85 \%$ de sa population totale (v. MEINANDER, 2008). La situation linguistique héritée de l'époque suédoise s'avéra très vite intenable; plus des quatre cinquièmes des habitants du pays ne pouvaient être relégués dans l'ignorance totale de la langue administrative. En outre, le finnois, étant donné qu'il avait été utilisé presque exclusivement dans des textes juridiques, administratifs et religieux, n'était pas apte à satisfaire les critères exigés d'une langue de culture. Pour ces raisons, un besoin impérieux de moderniser la langue finnoise se fit sentir parmi les premiers nationalistes qui avaient embrassé la cause du finnois. La modernisation de la langue était d'autant plus nécessaire qu'une partie influente de la population suécophone s'opposait assez violemment aux revendications linguistiques de la population majoritaire. Une des armes utilisées dans cette bataille contre le finnois était précisément le caractère peu normatif et vétuste de cette langue.

Jacob Judén (1781-1855), dans sa Grammaire finnoise (JUDEN, 1818), dit qu'il faut tirer la langue finnoise de l'oubli où elle a été ensevelie pour qu'elle puisse servir de langue de culture; sa décadence constitue un obstacle pour le développement intellectuel et matériel de la nation. Pour atteindre cet objectif, il faut pourtant que la langue devienne consciente d'elle-même :

Mais surtout, pour atteindre cet objectif, il est nécessaire que dans les circonstances actuelles, au moyen d'écrits intéressants et utiles, la langue réussisse à se reconnaître en ellemême et dans son lien avec la Nation, que les dialectes s'amalgament et que se forme une langue bien précise à l'oral et à l'écrit. Cela signifie: on doit écrire comme on parle. Mais d'après cette règle il y aurait autant de langues qu'il y a de dialectes; c'est ainsi que de chaque dialecte on ne doit prendre que ce qui le mieux s'adapte à la nature de la langue et peut contribuer à sa plénitude. [JUDEN $1818: 5$, traduit du suédois, JH]

Le problème qui se posait était de savoir quelle devait être la base de la nouvelle langue normative. La vieille langue littéraire, celle des textes religieux et

\footnotetext{
${ }^{1}$ En 1812, la Carélie finlandaise, cédée à la Russie dans les traités de paix de 1721 et 1743, fut restituée au Grand-Duché.
} 
administratifs de l'époque suédoise était basée principalement sur les dialectes de la région de la ville de Turku, siège de l'évêché de Finlande ${ }^{2}$ et capitale du pays jusqu'en 1812. Toutefois, au long des siècles, des éléments provenant de différents parlers finnois (surtout des parlers de l'Ouest) furent introduits dans la langue écrite. Même dans les textes d'Agricola, qui avait fait une partie de ses études à Viipuri dans l'est du pays, on trouve quelques tournures plus typiques des dialectes de l'Est. Selon HÄKKINEN (1994 : 436-451), il n'est pas toujours aisé de localiser le dialecte des premiers écrivains de langue finnoise. La division dialectale a pu changer au long de l'histoire et les études dialectologiques mieux documentées sont relativement tardives ; Porthan établit le premier la principale division en dialectes de l'Ouest et de l'Est. Cette analyse a été confirmée, avec de légères modifications, par de nombreuses études dialectologiques postérieures.

Les sources de la future langue normative étaient donc les suivantes (v. aussi HAVU \& KLIPPI, 2007) :

1. L'ancienne langue littéraire.

2. Les parlers régionaux.

3. Les langues étrangères (traductions, calques, etc.).

4. La structure interne de la langue.

En ce qui concerne l'influence des langues étrangères, elle a modifié le vocabulaire et bien des structures de la langue finnoise tout au long des siècles. Certaines catégories grammaticales et unités lexicales ont été introduites dans la langue grâce aux contacts linguistiques parfois même très anciens avec les langues indo-européennes (v. HÄKKINEN, 1994 : 452-494). Par exemple, les langues finnoougriennes sont à l'origine des langues typiquement synthétiques et agglutinantes, mais le finnois (et toutes les langues balto-fenniques) possède aussi des structures analytiques dont une partie est due à une influence indo-européenne, parfois directe, parfois indirecte. Dans ce travail, néanmoins, nous allons nous concentrer surtout sur l'évolution de la langue au $19^{\mathrm{e}}$ siècle, une époque où il y avait une grande effervescence linguistique et où la langue finnoise se transforma d'une langue éminemment rurale en une langue de culture.

Il faut pourtant mentionner un phénomène qui est lié au travail de modernisation de la langue, d'une part, et à l'influence étrangère, de l'autre. Il s'agit du purisme linguistique, tendance qui a été assez puissamment enracinée dans l'esprit des modernisateurs de la langue au $19^{\mathrm{e}}$ siècle et qui encore aujourd'hui est un principe idéologique du travail de l'institution normative officielle, Kotimaisten kielten keskus ${ }^{3}$ (Centre des langues nationales). Au $19^{\mathrm{e}}$ siècle, une partie importante du travail des codificateurs de la langue consistait à l'épurer des éléments étrangers. Ces éléments allogènes étaient plus visibles dans le lexique, et c'est précisément sur ce domaine que se concentrait l'attention des grammairiens. Pour ce qui est de la syntaxe, les contacts séculiers avec les langues indo-européennes avoisinantes (surtout le suédois, le russe, les langues baltiques,

\footnotetext{
${ }^{2}$ Transformé en archevêché en 1817.

${ }^{3}$ En Finlande, il y a deux langues nationales, le finnois et le suédois, officielles sur tout le territoire du pays. En outre, plusieurs langues minoritaires ont un statut officiel restreint (le same, le rromani, la langue des signes, etc., v. HAVU, 2009).
} 
l'allemand) avaient laissé des traces dans la morphosyntaxe et la syntaxe du finnois. Parfois ces influences s'étaient déjà profondément ancrées dans la langue et leur origine s'était perdue dans la conscience des locuteurs du finnois; c'est le cas, par exemple, des formes verbales composées (le parfait, le plus-que-parfait, etc.) dont l'origine étrangère n'était plus transparente. Certains phénomènes syntaxiques, tels que le passif agentif ${ }^{4}$, un calque du suédois, sonta ssez fréquents dans les textes de l'époque antérieure à 1809, mais il n'a jamais réussi à pénétrer le système de la langue spontanée et disparaît des grammaires du $19^{\mathrm{e}}$ siècle.

\section{La codification et la grammatisation du finnois au $19^{\mathrm{e}}$ siècle}

La plupart des grammaires parues au début du $19^{\mathrm{e}}$ siècle étaient des grammaires didactiques. La première grammaire vraiment importante et influente dusiècle mentionné fut celle de Reinhold von Becker, Finsk grammatik, (v. BECKER, 1824), écrite en suédois. La grammaire de von Becker est la première qui s'inspire en partie des dialectes de l'Est (lui-même était né à Kangasniemi, situé dans la province de Savo). Dans le deuxième numéro (paru en 1820) de la publication hebdomadaire Turun Wiikko-Sanomat (parue entre 1820 et 1831 dans la ville de Turku), dont il fut le rédacteur en chef, il constate que les Finnois, tout comme les autres nations, ont plusieurs dialectes et qu'il n'y a guère de municipalité qui n'ait sa propre façon de parler. Chacun pense que son dialecte est meilleur que les autres : mais seul celui qui connaît les dialectes de toutes les régions peut «savoir et dire où se trouve le parler le plus clair et qui, sans préférences aprioristiques, les a tous étudiés à fond ». Il ajoute :

Il est aisé de comprendre que du côté de la mer, où on parle souvent une langue étrangère et où il y a une population étrangère depuis longtemps établie, la langue finnoise est moins pure et plus déchue qu'à l'intérieur et au nord de la Finlande, moins exposés aux contacts avec des personnes parlant d'autres langues.[v. BECKER, Turun Wiikko-Sanomat, n. 2, le 15 janvier 1820 , p. 1, traduit du finnois, JH]

Comme il a été dit plus haut, von Becker cherche à introduire dans sa grammaire des variantes provenant des dialectes de sa région natale. Il considère le dialecte de Savo comme « den äldsta elle såsom grunddialekt (= le plus ancien ou comme un dialecte de base) » qui conserve mieux que les autres les traits les plus authentiques de la langue finnoise. Il reconnaît, pourtant, que les dialectes de l'Ouest ont été davantage utilisés dans la langue écrite et qu'ils sont par ce fait mieux connus dans tout le pays. Dans l'introduction, il constate que dans les dialectes de l'Ouest, malgré leur emploi à l'écrit pendant longtemps, il y a de nombreuses déformations de ce qu'on doit considérer comme autochtone. D'après l'auteur, les éléments provenant du dialecte de Savo enrichiraient et amélioreraient la langue normative en gestation :

\footnotetext{
${ }^{4}$ En finnois moderne, il n'y a pas de passif agentif mais seulement une forme impersonnelle du type syödään « on mange». Pendant l'époque suédoise, cependant, on utilisait souvent la construction präntätty Frenckelliltä « imprimé par Frenckell », où le participe passé est suivi d'un nom d'agent au cas ablatif. Il s'agit d'un calque du suédois tryckt av Frenckell.
} 
Il est facile de voir qu'en joignant ce qui est le plus régulier aux caractéristiques les plus avantageuses des dialectes principaux on pourra créer une langue écrite plus parfaite, et il est aisé de constater qu'une unification de ce genre peut se faire grâce aux textes supradialectaux qu'on trouve dans le pays. [v. BECKER, 1824 : VI, traduit du finnois par JH]

Cette citation met en évidence la volonté de certains des premiers grammairiens d'arriver à un compromis supradialectal concernant la langue normative. Or, von Becker ne réussit pas à doter sa grammaire d'une orientation normative. Il donne plusieurs variantes de bien des éléments morphologiques précisément à cause de sa volonté d'introduire des formes provenant des dialectes de l'Est à côté de formes plus traditionnelles de la vieille langue littéraire.

Le prestige des dialectes de l'Est s'est accru par la publication de l'œuvre qui allait devenir l'épopée nationale. Le Kalevala, dont la première version fut publiée en 1835 et la version définitive en 1849 (v. LÖNNROT, 1835 et 1849), fut un événement important pour l'identité nationale. L'épopée révéla au public cultivé la surprenante richesse et beauté des poésies populaires et fit découvrir les possibilités littéraires d'une langue jusqu'alors considérée comme primitive et rustre. Or, la tradition poétique orale avait été mieux conservée dans les régions orientales du pays dont les parlers différaient considérablement des dialectes de l'Ouest. Le compilateur du Kalevala, Elias Lönnrot, ${ }^{5}$ (disciple de von Becker), luimême originaire de l'Ouest, dut adapter certains phénomènes phonétiques et morphosyntaxiques typiques des dialectes de l'Est à des formes plus accessibles au public de l'ouest du pays (v. PUNTTILA\&ISSAKAINEN, 2003, p. 227-228). C'est ainsi que la langue du Kalevala donnait leurs lettres de noblesse aux dialectes de l'Est et fut ainsi un compromis entre les différents parlers finnois. La fragmentation dialectale produisit ce qu'on a l'habitude d'appeler la «lutte des dialectes ». Les sympathisants des dialectes de l'Ouest faisaient valoir une tradition littéraire vieille de presque trois siècles tandis que les militants des parlers orientaux, de leur côté, considéraient les dialectes de l'Est comme plus purs et plus authentiques, c'est-à-dire moins contaminés par les influences étrangères.

La réaction contre l'influence des dialectes de l'Est ne se fit pas attendre. Gustaf Renvall, qui était originaire de la zone de l'Ouest, commente la grammaire de Reinhold von Becker dans l'introduction de sa Grammaire (écrite vers 1820, mais publiée seulement en 1840), Finsk Språklära, Enligt den rena Vest-Finska, i Bokspråk vanliga Dialecten («Manuel de finnois, d'après le pur Dialecte de l'ouest de la Finlande, utilisé normalement dans la Langue littéraire »). Renvall justifie sa préférence pour la vieille langue écrite comme base pour la langue normative :

Mon principal souci a été de présenter la Langue finnoise comme elle a été utilisée à l'écrit pendant des siècles, et est familière à l'ensemble de la Nation et reconnue en vertu du temps écoulé. - Si la Grammaire de M. von Becker contient quelque erreur, c'est surtout dî au fait

\footnotetext{
${ }^{5}$ Le Kalevala est essentiellement l'œuvre de LöNNROT même si la plupart des vers avaient été recueillis par lui dans les zones rurales de l'Est de la Finlande et en Carélie orientale. D'après LÖNNROT, avec les milliers de vers recueillis, il aurait pu rédiger sept Kalevalas totalement différents.
} 
que, vu la préférence qu'il donne à son cher dialecte de Savo, il a dans plusieurs endroits essayé d'introduire dans la langue décrite des éléments provenant de ce dialecte, étrangers à la langue littéraire, et même erronés et mal formés, sans indiquer leur provenance; par où le vieux finnois a été habillé dans un costume multicolore avec des pièces de couleurs bigarrées cousues dessus. [RENVALL, 1840 : I, traduit du suédois par JH]

Les extraits que nous venons de présenter ci-devant montrent clairement les enjeux qui compliquaient considérablement cette première phase de la codification de la langue. Comme il n'existait aucune institution normative, la codification dut se mettre en marche sur la base d'initiatives individuelles. Il est évident que la langue littéraire utilisée à l'époque suédoise était irrémédiablement vieillie. Or, comme nous l'avons vu, les solutions proposées n'étaient pas uniformes ; certains grammairiens considéraient qu'il fallait moderniser la langue littéraire à partir des structures de la vieille langue littéraire, tandis que d'autres prônaient une sorte d'amalgame de dialectes.

Toute la première moitié du $19^{\mathrm{e}}$ siècle fut marquée par une lutte serrée pour fixer les critères de la normativisation de la langue. La position extrême fut celle de K. A. Gottlund (1796-1875), qui tout en étant de langue maternelle suédoise, adopta le dialecte de Savo comme son moyen d'expression en finnois. Il déclare que les Finnois ont une seule langue et que tous les dialectes font partie de cette langue. Il déplore la volonté d'en élever un seul pour servir de base à la future langue normative et dit que chacun doit avoir le droit d'utiliser son propre parler :

De quel droit élève-t-on un dialecte à la dignité de langue écrite et rabaisse-t-on tous les autres? [GotTLUND, 1831 : LXII, traduit du finnois par JH])

Le prestige personnel du compilateur du Kalevala, Elias Lönnrot, joua un rôle important dans le processus de codification de la langue. C'était un grand connaisseur des différents dialectes de la langue finnoise; son propre parler appartenait au domaine dialectal de l'Ouest, mais en quête des poésies traditionnelles, il avait parcouru pratiquement toutes les zones de l'Est du pays, jusqu'à la Carélie orientale. Dans l'introduction au recueil de poèmes de Paavo Korhonen, publié en 1848, il se plaint de la fragmentation dialectale du pays et surtout de ceux qui s'acharnent à la maintenir aussi dans la langue écrite. Il plaide pour une langue écrite unifiée :

En revanche, si l'on élabore, selon les lois fondamentales de la langue, une langue écrite unifiée, elle atteindra en dix ans la plénitude et la qualité auxquelles un seul dialecte mettrait plus d'un siècle à arriver. [KORHONEN, 1848 : 29, traduit du finnois par JH]

La «lutte des dialectes » s'apaisa pendant la seconde moitié du $19^{\mathrm{e}}$ siècle. Le prestige personnel de Lönnrot joua certainement un rôle dans le processus qui aboutit à un compromis de principe. Il fut établi que la base de la langue normative devait être la langue littéraire héritée de l'époque suédoise, enrichie de structures, de mots et d'expressions provenant de l'Est. En 1898, Arvi Jännes écrivait dans l'introduction à sa grammaire scolaire :

On peut dire que les dialectes populaires se rejoignent dans la langue littéraire au fur et à mesure que la civilisation et la littérature se répandent, modifient la langue orale et 
produisent une langue parlée soignée, commune à tous et libre de propriétés dialectales. [JÄNNES, $1898: 3$, traduit du finnois par JH]

La syntaxe de SETÄLÄ (1880) et la grammaire du même auteur (1898) ainsi que celle de JÄNNES (1881) fixèrent la norme. Des écrivains tels que Juhani Aho (1861-1921) et Minna Canth (1844-1897) furent les premiers dont la langue est conforme à la norme moderne.

L'accord de principe n'empêcha pourtant pas de nombreux débats concernant bien des détails grammaticaux et lexicaux. Un des débats les plus connus de la fin du $19^{\text {e }}$ siècle a été commenté en détail par LAITINEN (2007); il s'agissait de la forme correcte de la négation standard en finnois. ${ }^{6}$ Deux points de vue s'opposaient; le groupe autour de A.W. Ingman (secrétaire général du Comité linguistique de la Société de la littérature finlandaise) ${ }^{7}$ et celui dirigé par A. Ahlqvist (professeur de finnois de l'Université de Helsinki). Ingman défendait l'invariabilité de l'élément de négation (ex. 1), tandis qu'Ahlqvist soutenait qu'il fallait systématiquement le conjuguer d'après le sujet de la phrase (ex. 2). La particule $-k a /-k \ddot{a}$ s'ajoute à l'élément de négation dans des contextes de coordination (v. LAITINEN, 2007 : 178-179) :

\begin{tabular}{|c|c|c|c|c|}
\hline $\begin{array}{l}\text { 1.Emme } \\
\text { nég.1.pers.pl. }\end{array}$ & $\begin{array}{l}\text { puhu } \\
\text { parl- }\end{array}$ & $\begin{array}{l}\text { miehistä } \\
\text { homme.pl.elat. }\end{array}$ & $\begin{array}{l}\text { eikä } \\
\text { neg. } K A^{8}\end{array}$ & $\begin{array}{l}\text { vaimoista. } \\
\text { femme.pl.elat. }\end{array}$ \\
\hline 2.Emme & $\begin{array}{l}\text { puhu } \\
\text { parl- }\end{array}$ & $\begin{array}{l}\text { miehistä } \\
\text { homme.pl.elat. }\end{array}$ & $\begin{array}{l}\text { emmek } \ddot{a} \\
\text { neg.1.pers.pl.KA }\end{array}$ & $\begin{array}{l}\text { vaimoista. } \\
\text { femme.pl.elat. }\end{array}$ \\
\hline
\end{tabular}

Laitinen constate que ces débats, souvent violents et agressifs où les participants s'insultaient sans réserve, touchaient des structures fondamentales de la langue et que la raison sous-jacente des positions opposées était souvent la base dialectale des personnes impliquées. ${ }^{9}$ Or, il y avait également deux idéologies qui se heurtaient: d'un côté, les partisans d'une norme bien structurée et fondée sur une étude linguistique sérieuse, et de l'autre, les sympathisants d'une langue plus proche des formes langagières populaires. Comme il n'y avait pas d'institutions normatives, ces débats se déroulaient au sein d'associations civiles et souvent dans les colonnes des journaux ou des revues scientifiques. Les grammaires parues vers la fin du $19^{\mathrm{e}}$ siècle (surtout celle de E. Setälä), ainsi que le rapport du premier Comité linguistique du Sénat (1888), mirent fin à ces débats et optèrent pour une forme langagière moins influencée par des considérations dialectales et plus orientée vers la création d'une langue normative constituée sur la base d'une activité de recherche linguistique.

\footnotetext{
${ }^{6}$ La négation se construit au moyen d'un auxiliaire conjugué et d'une forme nominale invariable du verbe principal, par exemple syöd $\ddot{a}$ «manger » : en, et, ei, emme, ette, eivät + syö, « je ne mange pas, tu ne manges pas, etc. ».

${ }^{7}$ Ce comité (1868-1873) fut l'embryon des institutions normatives postérieures.

${ }^{8} \mathrm{La}$ particule $-k a /-k \ddot{a}$ s'attache normalement à un élément négatif et fonctionne comme une conjonction de coordination.

${ }^{9}$ Dans le dialecte d'Ingman, la négation était souvent invariable, tandis que dans celui d'Ahlqvist elle se comportait comme un verbe auxiliaire.
} 
La fixation de la norme était devenue urgente à cause de la promulgation du décret sur les écoles primaires (1866), qui jetait les bases de l'enseignement public. Les écoles primaires se généralisèrent assez lentement, de sorte que la dernière municipalité à avoir son école primaire fut Velkua en 1908. En revanche, la loi sur la scolarisation obligatoire entra en vigueur tard, après la proclamation de l'indépendance, en 1921. Dans les écoles primaires, une norme linguistique bien structurée était une nécessité pédagogique impérieuse, car pour la première fois les grandes masses rurales finnophones avaient accès à l'éducation, et pour assurer l'unité du pays, il fallait une langue commune.

Voici quelques exemples (parmi de très nombreux cas) des différences phonétiques et morphologiques entre les dialectes de l'Ouest et ceux de l'Est (la solution normative est en italique) :

\begin{tabular}{|c|c|c|c|}
\hline & Ouest & Est & \\
\hline élatif & talosa & talossa & «dans la maison » \\
\hline abessif & talota & talotta & «sans la maison » \\
\hline accusatif des pronoms personnels & minun & minut & «me / moi » \\
\hline diphtongues de la première syllabe & $\begin{array}{l}\text { nuari } \\
\text { tyä }\end{array}$ & $\begin{array}{l}\text { nuori } \\
\text { työ }\end{array}$ & $\begin{array}{l}\text { « jeune } » \\
\text { «travail } »\end{array}$ \\
\hline $3^{\text {ème }}$ pers. singulier de certains verbes & $\begin{array}{l}\text { saa } \\
\text { tulee }\end{array}$ & $\begin{array}{l}\text { saap, saapi } \\
\text { tuloo, tulloo }\end{array}$ & $\begin{array}{l}\text { «(il) reçoit » } \\
\text { «(il) vient » }\end{array}$ \\
\hline voyelle longue vs. diphtongue & $\begin{array}{l}\text { pää } \\
m a a\end{array}$ & $\begin{array}{l}\text { peä / piä } \\
\text { mua / moa }\end{array}$ & $\begin{array}{l}\text { « tête » } \\
\text { «terre » }\end{array}$ \\
\hline alternances phonétiques & $\begin{array}{l}\text { kala (nom.) } \\
\text { kalaa (part.) }\end{array}$ & $\begin{array}{l}\text { kala (nom.) } \\
\text { kallaa (part.) }\end{array}$ & $\begin{array}{l}\text { «poisson } ~ \\
\text { «du poisson } »\end{array}$ \\
\hline
\end{tabular}

Le lexique fut l'objet d'un énorme travail de modification. D'une part, il fallait créer des mots pour dénoter des concepts qui n'avaient pas été indispensables pour une langue rurale, et de l'autre, éliminer les mots tombés en désuétude ainsi que les emprunts étrangers pour rendre la langue plus moderne, plus «pure » et plus autochtone. Il y eut des dizaines de milliers de nouveaux lexèmes introduits dans la langue au $19^{\mathrm{e}}$ siècle et encore plus nombreux sont ceux qui n'ont pas été adoptés par les locuteurs (v. HÄKKINEN, 1994). Les limites du présent travail ne permettent pourtant pas une présentation exhaustive de l'évolution lexicale de la langue ; nous nous contentons de quelques observations concernant les principaux mécanismes employés pour moderniser le lexique.

\section{i) Élimination d'éléments étrangers.}

Gustaf Renvall, dans l'introduction de sa grammaire (RENVALL, 1840 : II) se plaint du «barbare galimatias » de certains locuteurs de Turku; d'après lui, il s'agissait d'une forme infectée de mots empruntés au suédois. Voici un de ses exemples avec la phrase correspondante en suédois et en finnois contemporain :

\begin{tabular}{|c|c|c|c|c|c|c|}
\hline $\begin{array}{l}\text { 3. } \mathrm{Se} \\
\mathrm{ce}\end{array}$ & $\begin{array}{l}\text { on } \\
\text { être.3.p. }\end{array}$ & $\begin{array}{l}\text { farlit } \\
\text { dangereux }\end{array}$ & $\begin{array}{l}\text { seilata } \\
\text { naviguer }\end{array}$ & $\begin{array}{l}\text { tormis } \\
\text { tempête.INES }\end{array}$ & $\begin{array}{l}\text { yli } \\
\text { sur }\end{array}$ & $\begin{array}{l}\text { haavin. } \\
\text { mer.GEN }\end{array}$ \\
\hline $\begin{array}{l}\text { Det } \\
\text { ce }\end{array}$ & $\begin{array}{l}\text { är } \\
\text { être.3.p. }\end{array}$ & $\begin{array}{l}\text { farligt } \\
\text { dangereux }\end{array}$ & & $\begin{array}{l}\text { segla } \\
\text { naviguer }\end{array}$ & $\begin{array}{l}\text { storm } \\
\text { tempête }\end{array}$ & $\begin{array}{l}\text { över havet. } \\
\text { sur mer.ART.déf }\end{array}$ \\
\hline
\end{tabular}




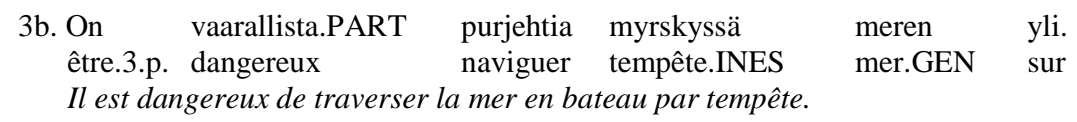

Dans la première phrase presque tous les mots sont des adaptations phonétiques des mots correspondants suédois. En outre, il y a des phénomènes grammaticaux calqués du suédois: i) l'emploi du sujet grammatical se; ii) l'emploi de l'adposition yli comme préposition et non comme postposition ce qui serait normal en finnois.

Pendant tout le $19^{\mathrm{e}}$ siècle et au début du $20^{\mathrm{e}}$, il y eut un travail constant d'élimination d'éléments allochtones.

\section{ii) Le système de dérivation et de composition}

Le développement lexical reposait sur deux caractéristiques structurelles de la langue : le système dérivatif particulièrement riche et flexible et la facilité de former des mots composés. Ces moyens ont été utilisés abondamment par les codificateurs de la langue afin de créer des mots pour des concepts qui n'en avaient pas. Dans les limites du présent travail, il n'est malheureusement pas possible de décrire en détail ces mécanismes de création lexicale à cause de leur grand nombre et de leur complexité. Nous nous en remettons à HÄKKINEN (1994: 407-435), qui présente un aperçu assez complet des principales lignes évolutives du lexique du finnois. Voici quelques exemples des mots fabriqués au $19^{\mathrm{e}}$ siècle :

- tiede « science »<tiedän « je sais »<tietää « savoir »

- taide « art» <taidan « je peux» <taitaa « pouvoir, être capable de»

- sanomalehti «journal $\gg<$ sanoma «message » + lehti «feuille »

D'après l'analyse de NIEMIKORPI (1991: 154), l'ancien dictionnaire normatif Nykysuomen sanakirja (Dictionnaire du finnois moderne, publié en 1951-1961) ${ }^{10}$ a 207256 entrées lexicales dont $65 \%$ sont des mots composés. Les lexèmes de base (non dérivés) représentent 8,6\% (17780 unités) et les mots dérivés des lexèmes de base $26,6 \%$ (55151 unités).

\section{iii) Les dialectes}

Comme c'est le cas des structures morphologiques, le lexique a aussi été marqué par la «lutte des dialectes». De nombreux mots provenant des dialectes de l'Est ont fait leur entrée dans la langue littéraire au $19^{\mathrm{e}}$ siècle. Cela a eu comme conséquence, d'une part, la disparition de certains mots qu'on trouve dans la vieille langue littéraire et de l'autre, la présence de doublets du type kesä / suvi «été », dont le premier est le mot normalement utilisé dans le finnois standard, tandis que le second a des connotations dialectales et poétiques. Parfois ces doublets gardent le même sens, comme p.ex. vasta / vihta « rameaux de bouleau avec lesquels on se fouette dans le sauna ».

\footnotetext{
${ }^{10}$ Le nouveau dictionnaire normatif est publié par Kotimaisten kielten keskus (Centre des langues nationales) et comprenden environ 100000 entrées. La différence entre le nombre d'entrées des deux dictionnaires s'explique en partie par le fait que le dictionnaire ancien incluait bien plus de dialectalismes et de mots dérivés et composés que le dictionnaire moderne.
} 


\section{iv) Les langues étrangères}

Nous avons déjà fait allusion à l'influence des langues étrangères et à la volonté d'éliminer une partie du vocabulaire directement emprunté aux langues voisines. Ce phénomène était plus fréquent lorsqu'il s'agissait d'objets ou de notions qui avaient trait à la vie quotidienne. En revanche, le domaine des concepts intellectuels comme p.ex. le vocabulaire scientifique conserve bien plus souvent des termes d'origine étrangère. L'évolution lexicale suit pourtant des sentiers insondables. Prenons comme exemple les mots liés à la vie académique. Universiteetti fut remplacé par yliopisto (yli- préfixe signifiant «supérieur»+ opisto «établissement de formation» <oppia «apprendre), mais akatemia reste inchangé. Les postes universitaires suivent les modèles internationaux, rehtori « recteur », professori «professeur », lehtori « lecteur», mais les institutions sont souvent (re)baptisées avec des noms formés au moyen d'éléments « autochtones »; fakulteetti a disparu, supplanté par tiedekunta «faculté » (<tiede « science » + kunta « communauté »). Pour ce qui est du domaine scientifique, les résultats sont hétérogènes. Par exemple, perinnöllisyystiede "génétique » (perinnöllisyys « hérédité »+ tiede «science ») est plus utilisé que genetiikka, mais on a opté pour biologia et kemia («biologie» et «chimie») au lieu de termes formés à partir d'éléments «autochtones». Les raisons qui expliquent l'entrée d'un mot dans l'usage général sont difficiles à élucider. Parfois, il peut s'agir de la sonorité du mot; akatemia est bien plus facile à prononcer que universiteetti.

\section{Conclusion}

Le finnois codifié est un artefact créé au $19^{\mathrm{e}}$ siècle. Nous avons cherché à décrire les grandes lignes de l'évolution du finnois au $19^{\mathrm{e}}$ siècle. Il s'agit d'une transformation très profonde de tout le système linguistique; le fait de transformer une langue rurale en une langue capable de fonctionner de manière satisfaisante dans tous les domaines de l'activité humaine a été un processus qui a affecté chaque sous-catégorie de la langue allant des structures grammaticales au lexique. Il va de soi que dans les limites de ce travail, nous n'avons pas pu aborder tous les détails de ce développement très complexe, mais nous espérons avoir pu montrer le caractère quelque peu artificiel de la langue normative. Il s'agit d'un compromis dont les composantes proviennent de sources hétérogènes. La base de la langue normative est la langue qu'on utilise à l'écrit dès les premiers textes publiés en finnois au $16^{\mathrm{e}}$ siècle, mais cette forme langagière a été modifiée et enrichie d'éléments provenant de sources variées : dialectes régionaux, langues étrangères, formation de nouveaux mots dérivés ou composés, etc. La langue normative n'a jamais été parlée de façon spontanée (sauf peut-être par une partie de la population suécophone qui a dû apprendre le finnois comme langue étrangère) mais son caractère supradialectal l'a rendue neutre et elle est généralement acceptée partout dans le pays. 
Une conséquence assez importante de l'évolution de la langue est l'écart entre le finnois parlé standard ${ }^{11}$ et la langue écrite. Cela est dû au fait que lorsque le travail de codification de la langue n'était pas encore achevé, les migrations vers les grandes villes, surtout Helsinki (la capitale du pays) avaient déjà commencé. La plupart des finnophones qui se sont installés à Helsinki au $19^{\mathrm{e}}$ siècle étaient originaires des zones de l'ouest de la Finlande de sorte que le finnois parlé standard est plus proche des dialectes occidentaux sans pour autant présenter de traits attribuables à un dialecte particulier. Ce finnois standard s'est développé parallèlement à l'évolution de la langue normative ; les différences existantes ne sont pas produites par une sorte de «dégradation» de la langue normative, mais sont des formes qui perpétuent les traits dialectaux de la langue standard formée à partir de la seconde moitié du $19^{\mathrm{e}}$ siècle.

Pour conclure, nous donnons un exemple des différences morphologiques qui met en évidence l'écart entre les deux formes langagières :

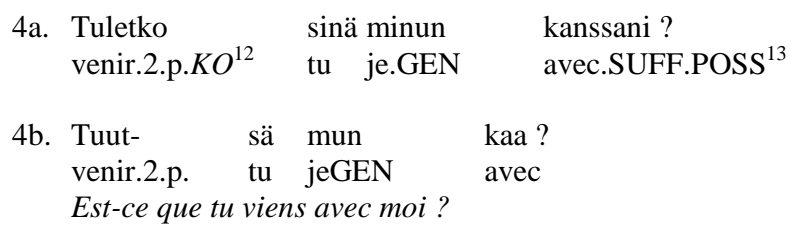

Cet état de choses rend difficile l'enseignement du finnois comme langue étrangère. Pour un non-natif, il n'est pas aisé de distinguer les registres et leur emploi correct selon les différentes situations interactionnelles.

La codification du finnois est un processus qui illustre bien le fait qu'une langue, considérée comme inepte et primitive, peut atteindre le statut de langue de culture et constituer l'élément central de l'identité nationale. Ce genre de situations n'est pas rare aujourd'hui. Au Paraguay, par exemple, l'espagnol est la principale langue administrative et d'enseignement bien que le guarani soit la langue parlée par $85 \%$ de la population comme première langue apprise. Le discours sur la question linguistique est presque identique à celui qui se déroulait en Finlande il y a 200 ans; pourquoi chercher à promouvoir le guarani, langue «primitive », vu que l'espagnol est une langue internationale. Rien de nouveau sous le soleil.

\section{BIBLIOGRAPHIE}

Il existe un très grand nombre d'études sur les thématiques abordées dans le présent travail. La plupart de ces études sont écrites en finnois et, par conséquent, difficilement accessibles à un public international.

\footnotetext{
${ }^{11}$ Par «finnois parlé standard » nous entendons une forme langagière neutre généralement utilisée dans une conversation normale presque partout dans le pays.

${ }^{12} \mathrm{~L}$ 'élément $-k o /-k \ddot{o}$ est la marque de l'interrogation.

${ }^{13}$ La langue standard emploie beaucoup moins les suffixes possessifs que la langue normative.
} 
BECKER Reinhold v. (1824), Finsk grammatik, Åbo, Bibel-sällskapets tryckeri.

DESPAGNET Frantz (1901), La Question finlandaise au point de vue juridique, Paris, Librairie de la Société du recueil général des lois et des arrêts.

Finlande et Russie; délibération internationale de Londres (1911), Paris, A. Pedone.

GotTLund Carl Axel (1831), Otava eli suomalaisia huvituksia, Stockholm, M.G.Lundberg.

HAVU Jukka (2009), 'Les Langues minoritaires parlées en Finlande', in : HAVU, Eva (éd.), Langue et identités finlandaises, Cahiers de la Nouvelle Europe, Paris, L'Harmattan, pp. 117-130.

HAVU Jukka [sous presse], 'La Géographie linguistique de la Finlande', in: CARAYOL, Martin; PELTOLA, Rea: Singularités, pluralités - Identités linguistiques et littéraires en Finlande. Presses Universitaires de Caen, pp. 2545.

HAVU Jukka, KLIPPI, Carita (2007), 'Finnois, Finlande, une langue, une nation. Contexte et corpus de codification du finnois moderne', Histoire, Épistémologie, Langage XXVIII, 2 «Hyperlangues et fabriques de langues», p. 81-120.

Hentilä Seppo, Jussila Osmo, NeVAKIVI Jukka (1999), Histoire politique de la Finlande - XIX $X^{e}-X X^{e}$ siècle, éditions Fayard.

HÄKKINEN Kaisa (1994), Agricolasta nykykieleen. Juva, WSOY.

JÄNNES Arvi (1898), Suomen kielioppi ( ${ }^{{ }^{e ̀ m e}}$ édition), Helsinki, Weilin \& Göös.

JUDÉN Jacob (1818), Försök till utredande af Finska Språkets Grammatik, Wiborg, And. Cederwaller.

JUSLENIUS Daniel (1745), Suomalaisen Sana-Lugun Coetus/Fennici Lexici Tentamen, Stockholm, Lars Salvius.

JutikKala Eino (1978), Histoire de la Finlande, Neuchâtel, Éditions de la Baconnière.

JÖNSSÖN-KORHOLA Hannele, LINDGREN, Anna-Riitta (2003), Monena suomi maailmalla - Suomalaisperäisiä kielivähemmistöjä, Helsinki, Suomalaisen Kirjallisuuden Seura.

KORHONEN Paavo (1848), Wiisikymmentä runoa ja kuusi laulua, Helsinki, Simeliuksen leski.

LAITINEN Lea (2007), "Kieltosana ja kieletär: Yhden kielikiistan kulku ja ideologiat", dans: Katja HuUMO, Lea LAITINEN, Outi PALOPOSKI (éds.), Yhteistä kieltä tekemässä - näkökulmia suomen kirjakielen kehitykseen 1800luvulla, Helsinki, Suomalaisen Kirjallisuuden Seura.

LE CALlOC'H Bernard (2010), Histoire de la Finlande, Paris, Éditions Glyphe.

LÖNNROT Elias (1835), Kalewala taikka Wanhoja Karjalan Runoja Suomen kansan muinoisista ajoista, Helsinki, Frenckell.

LÖNNROT Elias (1849), Kalevala, Helsinki, Suomalaisen kirjallisuuden seura.

Martinus Matthias (1689), Hodegus Finnicus, Omnibus hanc linguam discere cupientibus valde utilis. Eller Finsk Wägwijsare/Allom dem/som Finska Tungmåhlet ex fundamento declinera, conjugera, construera och elliest lära willia/ganska nyttig, Tavast-Finnone, Holmiae. 
MEINANDER Henrik (2008), Kekkografi och andra historiska spånor, Helsingfors, Söderströms.

NIEMIKORPI Antero (1991). Suomen kielen sanaston dynamiikkaa, Acta Wasaensia 26. Kielitiede 2. Vaasa, Université de Vaasa

Petraeus Aeschillus (1649), Linguce Finnicae brevis institutio, Åbo, Peder Eriksson Wald.

PuntTILA Matti, ISSAKAINEN, Touko (2003), « Kalevala, kansanrunous ja kirjakieli », Virittäjä 2, p. 226-245.

RANTANEN Päivi (1998), Suolatut säkeet - Suomen ja suomalaisten diskursiivinen muotoutuminen 1600-luvulta Topeliukseen, Suomalaisen Kirjallisuuden Seuran toimituksia 690, Jyväskylä, Gummerus Oy.

ReNVALl Gustaf (1840), Finsk Språklära, Enligt den rena Vest-Finska, $i$ Bokspråk vanliga Dialecten, Åbo, Christ. Ludv. Hjelt.

SETÄLÄ Emil Nestor (1880), Suomen kielen lauseoppi. Oppikirjan koe, Helsinki, K. E. Holm.

VHAËL Bartholdus (1733), Grammatica Fennica, Aboæ, Johan Kiämpe. 